\title{
A Reputation-Based Grid Information Service
}

\author{
J. H. Abawajy and A. M. Goscinski \\ Deakin University, \\ School of Engineering \& Information Technology, \\ Geelong, Victoria 3217, Australia
}

\begin{abstract}
In a large-scale wide-area system such as the Grid, trust is a prime concern. The current generation of grid information services lack the ability to determine how trustworthy a particular grid service provider or grid customer is likely to be. In this paper, we propose a grid information service with reputation management facility and its underlying algorithm for computing and managing reputation in serviceoriented grid computing. Our reputation management service is based on the concept of dynamic trust and reputation adaptation based on community experiences. The working model and functionality offered by the proposed reputation management service is discussed.
\end{abstract}

\section{Introduction}

Grid computing aims to enable resource sharing and coordinated problem solving in dynamic, multi-institutional virtual organizations (VO) [10. In Grids, federation is typically motivated by a need to access resources or services that cannot easily be replicated locally 9. Grids are currently evolving towards a service-oriented computing by adopting Web services-based and WSRF technologies [14 4]. An example is the the latest Globus Toolkit (i.e., GT4) 9] 3], which is based on the Open Grid Services Architecture (OGSA) [11. A service in a service-oriented computing can be computers, storage, data, networks, or sensors.

The Grid Information Service (GIS) is one of the main services offered by Grids. GIS maintains information about hardware, software, services and people participating in a VO. Moreover, GIS provides fundamental mechanisms for service advertisement and service discovery [10. It allows service providers to publish their offerings while grid customers can use services provided by GIS to discover services that meet their QoS requirements. However, when integrating services across multiple partners that form a VO, trust issues become significant. For example, how can grid service customers choose a trustworthy service provider without prior interaction? Similarly, service providers are commonly concerned if the customer is legitimate and able to pay for services rendered. As customers and service providers are increasingly demanding trustworthy paid services in the digital economy [12, the answer to these questions are important. However, the current generation of grid information services lack the ability to assist service providers (users) in determining how trustworthy a specific customer (provider) is likely to be. 
In this paper, we address this problem and propose a reputation-enabled grid information service that maintains a dynamic reputation metric such that grid customers can select a reliable service provider to transact with. Similarly, the proposed approach enables the service providers to tailor payment methods to a particular client based on credit rating of customers. The proposed reputation management service is based on the concept of dynamic trust and reputation adaptation based on community experiences. The feedback from the participants is analyzed, aggregated with feedback received from other members and made publicly available to the community in the form of service provider and customer reputation profiles. We present the underlying algorithm for computing and managing reputation in service-oriented grid computing environments.

The rest of the paper is organized as follows. In Section 2, we provide a short overview of the current research efforts that form the basis of our work. The working model and functionality offered by grid directory services and their limitations with respect to the questions at hand are briefly discussed. In Section 3 , we propose a new framework for managing reputation in service-oriented grid computing and discuss its underlying architecture. In Section 4, we discuss how the reputation and credit values for service providers and consumers are computed. We summarize future work and conclude our work in Section 5 .

\section{Problem Statement and Related Work}

\subsection{Problem Statement}

Reputation is of core importance when consumers and providers engage in situations which they perceive as risky. In this paper, reputation refers to the value we attribute to a specific entity including agents (e.g., brokers), services, and persons in the service grid, based on the trust exhibited by it in the past. In a typical Grid scenario users are interested in identifying possible candidate services through grid information service in a similar manner to an online shopping site [5].

There are many information services enabling service providers to publish their services while at the same time allow customers discover services of interest from among a large pool of potentially interesting services (e.g., [1] 8] and [14). As far as we know, existing GIS have not yet addressed the issue of integrating reputation into their resource discovery algorithms. Thus, GIS users optimistically assume all trading partners are trustworthy. As a result, both service providers and consumers are forced to assume all the risks associated with a particular service and resources obtained through the existing GISs.

However, customers are interested in selecting a reliable service provider to transact with. Similarly, the service providers want to tailor payment methods to a particular client based on credit rating of customers. Reputation-enabled information service can address these concerns. Reputations hold entities accountable for their actions and deter bad behavior [12. Entities that engage in good behavior build a positive reputation and people will continue to interact with it. Such a system encourages individuals to act in a trustworthy manner [7]. Since GISs provide automated resource and services discovery, we believe that 
the reputation service should be loosely integrated with the GIS such that desired services are automatically discovered by GIS and then ranked based on the reputation they obtain by the reputation services.

\subsection{Related Work}

Numerous information services that enabling service providers to advertise their services as well as allow customers discover services of interest. For example, the Universal Description, Discovery and Integration (UDDI) 1] defines a standard for enabling businesses to publish or discover Web services. Several efforts to extend UDDI for grid service discovery is underway (e.g., [13). The Monitoring and Discovery System (MDS) 8 is the information services component of the Globus Toolkit [9] and provides information about the available resources on the Grid and their status. The latest version of MDS includes Web Service Resource Framework (WSRF) based implementations of the Index Service, a Trigger Service, WebMDS (formerly known as the Web Service Data Browser) and the underlying framework, the Aggregator Framework. A market-oriented grid directory service (GDS) for publication and discovery of grid service providers and their services is discussed in 14 .

Several examples of feedback mechanisms are already being used in a number of well-known online communities, such as eBay [2]. A growing body of empirical evidence seems to indicate that these systems have managed to provide remarkable stability in otherwise very risky trading environments 12 . We envision a reputation service similar to the online shopping [2] for service-oriented grid computing. However, reputation requirements of the grid systems differ from that of the online shopping reputation system. For example, in eBay [2, buyers reputations matter substantially less, since sellers can hold goods until they are paid. Moreover, even if sellers wished to rely on buyers reputations it would do little good, since it is not possible to exclude buyers with bad reputations from ones auction. Also, there is a potential difficulty in aggregating and displaying feedback so that it is truly useful in influencing future decisions about who to trust. Finally, the reputation systems are for single context. In contrast, contexts in Grids can be numerous.

Recently, trust has been receiving increasing attention in grid computing community [5] [6]. The focus, however, is on grid security system to formulate trust enhanced security solutions. In contrast, we are interested in reputation enhanced grid information services. To the best of our knowledge, there is a lack of reputation information about services, resources, service providers and service users in existing grid information services. This motivated us to design a reputation service for augmenting grid information services.

\section{Reputation-Based Grid Information Service}

In this section, the proposed reputation-enabled grid information service infrastructure is discussed. We assume that once registered, both service providers and customers will have unforgeable identities. Unforgeable identities are usually generated by a trusted system entity and given to new users as they join. We 


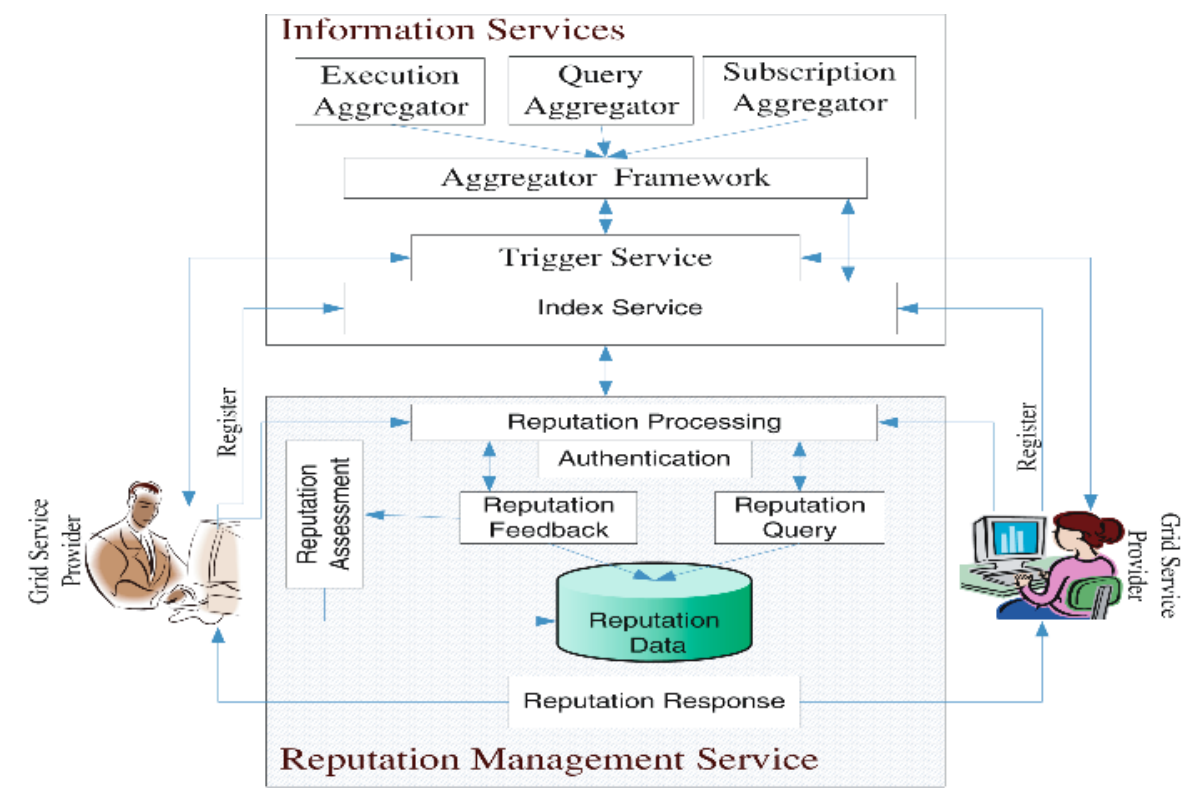

Fig. 1. Reputation-based Grid Information Service

also assume that there is no way a single user can generate multiple identities to pretend as several distinct service provider or customer or both in the network.

The overall design of the proposed reputation-based grid information service is shown in Fig. 1. The system is composed of information service and reputation management service components. Note that upon registration, the service providers (consumers) are informed to maintain a minimum trust level $\left(\Phi_{\text {min }}\right)$ at all times. By approximately automating word-of-mouth reputation for grid market environments, the proposed reputation-enabled grid information service infrastructure can provide a service that mitigates misbehavior while imposing a minimal cost on the well-behaved users.

The proposed approach uses credit score and reputation score to manage creditworthiness and trustworthnes of the GSC and GSP respectively. Reputation score have proved to be a great asset for both online shoppers and sellers to establish trust. Similarly, credit scoring is an important tool used by todays financial institutions to assess the creditworthiness of prospective customers and to monitor customer credit ratings. Credit scoring enables service providers to offer differentiated products and benefits based on credit information from customers.

\subsection{Information Service}

The information service component is the same as the conventional grid information services such as the MDS [8]. It is composed of a suite of web services including Index service, Trigger service and Aggregator framework, which is 
used by the Index and Trigger services. The central role of information services is to provide efficient resource registration, monitoring and resource discovery including subscription/notification. It maintains information about the resources available within grid computing such as hosts, clusters, switches, routers, links, services, sensors, available software, and services. As the focus of this paper is on the reputation management service, we will not discuss the information service components in the rest of the paper.

\subsection{Reputation Management Service}

The main goal of the reputation management service ( $R M S)$ component is to assist grid service providers and customers to confidently select trading partners. It collects and processes reputation data from the service providers and consumers. It also maintains a dynamic reputation metric for its community and provides several mechanisms for manipulating the reputation data in the repository with the objective of automating word-of-mouth reputation for grid market environments. The functionality of the RMS is encapsulated in five components: Reputation Processing, Reputation Query, Reputation Feedback, Reputation Response, and Reputation Assessment.

The Reputation Feedback allows service provider and consumer to rate each other only after they are engaged in service trading Each feedback consists of a rating: positive, negative, or neutral. Once the raw reputation data is collected from the service providers and consumers, the Reputation Assessment component calculates a reputation score for the service provider and consumer taking into account several factors including the reputation of the feedback provider and the cost of the service. The reputation query subcomponent allows service providers and consumers to access the reputation information after being authenticated to have been registered users.

The main responsibility of a response manager is to sanction entities in the service grid that are not behaving consistently and who break trust relations. The action that the the response manager takes when a service provider (consumer) reputation level falls below $\Phi_{\text {min }}$ is configurable. For example, it can be configured to send the service provider (consumer) a simple warning or immediately eject it from the network for a period of time or permanently banned. To reenter the system, the service providers (consumers) would need to acquire a new valid identifier, which may be costly or impossible. The shaded part of Fig. 1 shows the interaction between the RMS subcomponents as well as with the other system components (e.g., service providers and consumers).

\section{Reputation Assessment}

Reputation and credit data are collected from the grid service customer (GSC) and the grid service providers (GSP) after the completion of the service. The data is then processed to be represented as credit score and reputation score respectively. Reputation score provides a way of assigning quality or value to a 
service. In this work, reputation and credit of an entity is represented using a value in the interval between -1 and 1 . As this value approaches -1 , the entity becomes increasingly distrusting and conversely, as it approaches 1 the entity has complete or blind trust. We now discuss how the reputation score is computed for an entity $P_{i}$ at time t.

Note that service contexts (i.e., c) in grid computing can be numerous, varying from executing jobs, storing information, downloading data, and using the network. The overall cumulative reputation score, $R\left(P_{i}, t, c\right)$, for context $c$ of $P_{i}$ service provider at time $t$ is defined as follows:

$$
R\left(P_{i}, t, c\right)=\min \left(\left(R_{n e w}\left(P_{i}, t, c\right), 1.0\right)\right.
$$

where $t$ is the current time and $R_{\text {exist }}\left(P_{i}, t-1, c\right) \geq 0$ is the reputation score of an entity $P_{i}$ for context $c$ at time $t-1$.

The parameter $R_{n e w}\left(P_{i}, t, c\right)$ in Eq. 1 is the sum of the previous and the current reputation score and is computed as follows:

$$
R_{\text {new }}\left(P_{i}, t, c\right)=R_{\text {current }}\left(P_{i}, t, c\right)+R_{\text {exist }}\left(P_{i}, t-1, c\right)
$$

where the parameter $R_{\text {current }}\left(P_{i}, t, c\right)$ is computed as follows:

$$
R_{\text {current }}\left(P_{i}, t, c\right)=\text { Feedback }(c, t)+\text { Price }_{t}(c)+\text { Credibility }_{t}\left(P_{i}, t, c\right)
$$

From Eq. 3, we observe that there are three factors contributing to the current reputation score of a given service provider (consumer): (1) feedback provided by the rater (i.e., $\left.\Theta_{f}\right) ;(2)$ the price of the service (i.e., Price $_{t}(c)$ ); and $(3)$ the credibility of the rater (i.e., $\left.\operatorname{Credibility~}_{t}\left(P_{i}, t, c\right)\right)$.

To determine the contribution of the Rater Feedback to the current reputation score, let $f \in\left\{\right.$ positive, negative, neutral\} be $P_{i}$ 's feedback at time $t$ (i.e., right after the completion of the latest service) for context c. Note that a service provider, $P_{i}$, will only be allowed to supply the reputation data when the payment method used involves credit (e.g., pay-as-you-go and pay-afteruse). If pay-before-use is used, then the consumer will be automatically given $f=$ positive).

$$
\text { Feedback }(c, t)=1-\alpha^{f}
$$

Following the completion of the service, both the service provider and the consumer rate the quality of service as positive (i.e., $f=1$ ) or as negative (i.e., $f=-1$ ) or as neutral (i.e., $f=0$ ). Based on the value of $f$, the contribution of the feedback to the current reputation score is computed as in Eq. 4 where $0 \leq \alpha_{t} \leq 1$

The Price $_{t}(c)$ in Eq. 3 denotes the contribution of the service cost to the current reputation score and is given as follows:

$$
\operatorname{Price}_{t}(c)=1-e_{t}^{-\lambda \aleph}
$$

where $\aleph$ is the price paid for the service. Intuitively, a GSP that defects on one $\$ 100$ transaction should have a lower reputation than one who defects on two or 
three $\$ 1$ transactions. It is also important that one large transaction does not elevate the rate of an entity from nothing to complete or blind trust level. Thus, we constrain the range $\lambda$ can take as follows:

$$
\text { Feedback }=\left\{\begin{array}{l}
\text { negative } \lambda \geq 0.01 \\
\text { positive } \lambda \geq 0.0001 \\
\text { nuetral } \lambda \geq 0.00001
\end{array}\right.
$$

This avoid one of the main problems with existing reputation systems in which service providers build up a high positive reputation based on many small transactions and then defraud one or more buyers on a high-priced item.

The trustworthiness of the feedback provider (i.e., Credibility $\left.{ }_{t}\left(P_{i}, t, c\right)\right)$ contribution in the calculation of the current reputation score is given as follows:

$$
\text { Credibility }_{t}\left(P_{i}, t, c\right)=\left\{\begin{array}{lr}
0 & 0 \leq T \leq 1 \\
\bar{N}^{-\left(\frac{\bar{N}}{\beta \cdot T}\right)}-1 & \text { Otherwise }
\end{array}\right.
$$

where $\bar{N}$ denotes the total number of negative feedbacks so far received by $P_{i}$ and $T$ denotes the total number of feedbacks a grid service provider has so far accumulated and $\beta=\frac{\bar{N}}{T}$ while $T$ is given as follows:

$$
T=\sum F \text { Feedback }\left(P_{i}, c, \pm\right)+\mu \sum \text { Feedback }\left(P_{i}, c, \sim\right)
$$

where $\sum$ Feedback $\left(P_{i}, c, \pm\right)$ is the sum of positive and negative feedbacks while $\sum$ Feedback $\left(P_{i}, c, \sim\right)$ is the sum of neutral feedbacks received so far by $P_{i}$ respectively. We believe that the neutral feedbacks should only contribute a portion to the reputation score of $P_{i}$. This is because, in real life, humans tend to take neutral position when faced with two alternatives for which they do not want to cast votes to. Thus, we use $\mu$ such that $=0 \leq \mu \leq 1$ to capture the real life scenario.

\section{Conclusion and Future Direction}

Although there are variety of information services that could potentially enable grid users discover services and resources, none of these middleware services provide a direct or indirect means of discovering reputable grid services and resources. In this paper, we have introduced a reputation management support in grid information service. We believe that reputation serves as an important metric to avert the usage of under provisioned and malicious resources with the help of community feedback. As a future work, an important issue to be addressed is that if the resource selection decisions are contingent only on the reputation, severe load imbalance can occur in a large-scale grid computing with some dominant resources. Therefore, a mechanism for balancing performance against reliability will be investigated. Another future issue to be looked at is how to elicit feedback from the participants. We are currently performing analysis of reputation assessment algorithm. Finally, in the proposed approach, 
a service provider (consumer) can be immediately ejected it from the network for a period of time or permanently banned. A mechanism for allowing service providers (consumers) to reenter the system would be needed, which we are currently working on.

Acknowledgments. The first author would like to thank Maliha Omar for all the helps.

\section{References}

1. http://www.uddi.org.

2. http://www.ebay.com.

3. Globus toolkit. http://www.globus.org.

4. J. H. Abawajy, editor. Grid Accounting Service Infrastructure for Service-Oriented Grid Computing Systems. Springer-Verlag, 2005.

5. B. K. Alunkal, I. Veljkovic, G. von Laszewski, and K. Amin. Reputation-based grid resource selection. In Proceedings of AGridM 2003, 2003.

6. F. Azzedin and M. Maheswaran. Towards trust-aware resource management in grid computing systems. In Proceedings of CCGRID'02, page 452, 2002.

7. S. Ba and P. Pavlou. Evidence of the effect of trust building technology in electronic markets: Price premiums and buyer behavior. MIS Quarterly, 11(26(3)), 2002.

8. K. Czajkowski, S. Fitzgerald, I. Foster, and C. Kesselmand. Grid information services for distributed resource sharing. In Proceedings of the HPDC-10, 2001.

9. I. Foster. Globus toolkit version 4: Software for service-oriented systems. In Proceedings of IFIP International Conference on Network and Parallel Computing, pages $2-13,2005$.

10. I. Foster and C. Kesselman, editors. The grid: blueprint for a new computing infrastructure. Morgan Kaufmann Publishers Inc., San Francisco, CA, USA, 1999.

11. I. Foster, C. Kesselman, J. Nick, and S. Tuecke. The physiology of the grid: An open grid services architecture for distributed systems integration. IEEE Computer, 35(6), 2002.

12. P. Resnick and R. Zeckhauser. Trust among strangers in internet transactions: Empirical analysis of ebay's reputation system. In M. R. Baye, editor, The Economics of the Internet and E-Commerce. Advances in Applied Microeconomics, volume 11. JAI Press, Greenwich, CT, 2002.

13. B. Sinclair, A. Goscinski, and R. Dew. Enhancing uddi for grid service discovery by using dynamic parameters. In ICCSA (3), pages 49-59, 2005.

14. J. Venugopal and R. Buyya. A market-oriented grid directory service for publication and discovery of grid service providers and their services. Journal of Supercomputing, 2005. 